\title{
Clinical and Microbiological Spectrum of Clostridial Myonecrosis from a Tertiary Care Centre
}

\section{Sukanya Sudhaharan ${ }^{1}$, Kanne Padmaja ${ }^{2 *}$, Padmasri Chavali ${ }^{3}$ and Vijay Dharma Teja ${ }^{4}$}

${ }^{1}$ Assistant Professor, Department of Microbiology, Nizams Institute of Medical Sciences, Hyderabad, Telangana, India

${ }^{2}$ Associate Professor, Department of Microbiology, Nizams Institute of Medical

Sciences, Hyderabad, Telangana, India

${ }^{3}$ Lab Technician, Department of Microbiology, Nizams Institute of Medical Sciences,

Hyderabad, Telangana, India

${ }^{4}$ Professor and HOD, Department of Microbiology, Nizams Institute of Medical

Sciences, Hyderabad, Telangana, India

*Corresponding Author: Kanne Padmaja, Associate Professor, Department of Microbiology, Nizams Institute of Medical Sciences, Hyderabad, Telangana, India.
Received: June 22, 2020

Published: August 26, 2020

(C) All rights are reserved by Kanne

Padmaja., et al.

\begin{abstract}
Introduction: Infections due to Clostridium spp is most often seen in traumatic injuries, surgery, malignancy and underlying immunocompromised conditions. Gas gangrene, the clinical manifestation of Clostridium spp is a diffuse necrotizing infection of deep soft tissues. The infection can develop rapidly following any trauma that compromises the blood supply and is characterized by high mortality. Early Identification of the signs and symptoms in these patients would help in the management and reduce mortality associated with this infection. In this review we presented a series of cases with infection due to Clostridium spp after trauma.

Materials and Methods: Twelve cases of Clostridium perfringens infections were analysed from our Institute prospectively during the period of 2012 - 2019 (duration of 8 years). The tissue samples from the cases were subjected to direct Gram stain and further inoculated aerobically on Chromogenic agar and 5\% sheep blood agar. Anaerobic inoculation was done on 5\% sheep blood agar and incubated into anaerobic pouch.

Results: The median age of the patients were 31 years. All the patients were males. The patients had sustained injury due to road traffic accident (RTA). 5/12 patients had Grade 3 compound fracture of leg, 1/12 patient had injury extending up to thigh, 5/12 patients had crush injury of lower limb, 1/12 patient had crush injury of upper limb.

Escherichia coli was the predominant aerobic organism isolated in $7 / 12$ of the patients. The patients were treated surgically with below knee amputation in 10/12, below elbow amputation in $1 / 12$, above knee amputation in $1 / 12$ of patients. All the patients were treated with intravenous penicillin G10 U/day. Eleven patients were discharged in stable condition with advise to follow up. The patient with above knee amputation succumbed to the infection in spite of the treatment.

Conclusion: From this study we conclude that early clinical suspicion and microbiological diagnosis helps in the proper management of the patients. Careful and adequate treatment along with antibiotics and all other supportive treatments should be instituted immediately will avoid further deterioration and reduce the mortality in these patients
\end{abstract}

Keywords: Toxins; Deep Tissue Infection; Virulent; Muscle Necrosis; Trauma

Citation: Kanne Padmaja., et al. "Clinical and Microbiological Spectrum of Clostridial Myonecrosis from a Tertiary Care Centre". Acta Scientific Microbiology 3.9 (2020): 47-52. 


\section{Introduction}

Infections due to Clostridium spp is most often seen in traumatic injuries, surgery, malignancy and underlying immunocompromised conditions [1]. Gas gangrene refers to a life threatening, toxin mediated clinical manifestation of Clostridium spp, that leads to a diffuse necrotizing infection of deep soft tissues [2]. This can be divided into traumatic and spontaneous types [3]. Traumatic, resulting from penetrating injury and inoculation of muscle tissue with Clostridium perfringens, an anaerobic bacteria found in sewage and soil. Atraumatic or spontaneous, most often arising from hematogenous spread of Clostridium septicum from the gastrointestinal (GI) tracts of neutropenic patients like leukemia, diabetes mellitus or those with GI malignancies like carcinoma of colon or rectum [3]. The infection can develop rapidly following any trauma that hinders or limits the blood supply to the area and is characterized by high mortality [4,5]. Early Identification of the signs and symptoms in these patients would help in the management and reduce mortality associated with this infection. Empiric treatment with effective antimicrobial agents is another crucial part of the management of the patients with gas gangrene. In this review we present a series of cases with infection due to Clostridium perfringens after trauma.

\section{Materials and Methods}

A total of twelve cases of Clostridium perfringens infections were analysed prospectively during the period of $2012-2020$ (8 years) from our institute. The tissue samples from the cases were subjected to direct Gram stain which showed Gram positive bacilli. The sample was further inoculated aerobically in Chromogenic agar, 5\% sheep blood agar. Anaerobic inoculation was done on 5\% sheep blood agar and incubated into anaerobic pouch (Gen Bag, Biomeriux).

\section{Results}

The median age of the patients were 31 years. Age of the patients ranged from 30 - 65 yrs (Table 1). All the patients were males. The patients had sustained injury due to road traffic accident. 5/12 patients had Grade 3 compound fracture of leg, 1/12 patient had injury extending up to thigh, $5 / 12$ patients had crush injury of lower limb, 1/12 patient had crush injury of upper limb.

Escherichia coli was the predominant organism isolated in aerobic culture (Figure 1). Mixed infection of aerobic organisms was observed in 4/12 samples (Table 2). Polymicrobial growth was

\begin{tabular}{|l|c|c|c|}
\hline $\begin{array}{c}\text { S. } \\
\text { No }\end{array}$ & Age group & No of patients & Percentage \\
\hline 1 & $20-30$ & 5 & 41.6 \\
\hline 2 & $30-40$ & 3 & 25 \\
\hline 3 & $40-50$ & 2 & 16.6 \\
\hline 4 & $50-60$ & 2 & 16.6 \\
\hline
\end{tabular}

Table 1: Distribution of age group of patients.

observed in 1/12 of the cultures. All the patients were managed by both surgical and medical treatment. The patients were treated surgically with below knee amputation (10/12), below elbow amputation (1/12), above knee amputation in one patient (1/12).

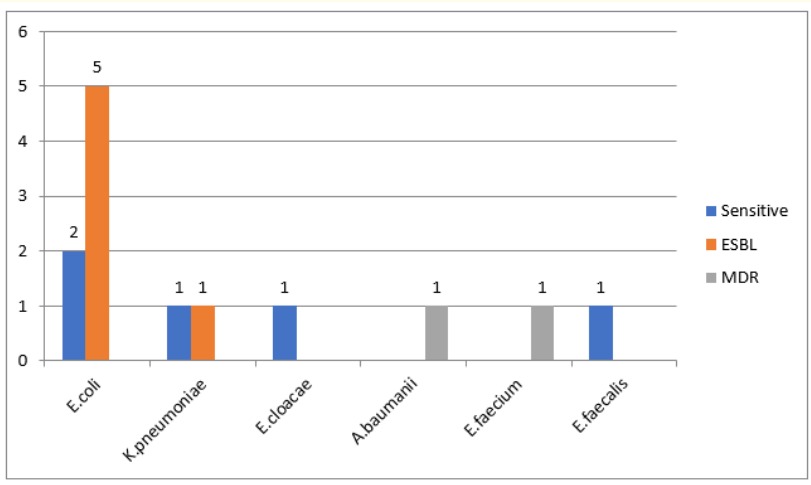

Figure 1: Graph showing aerobic organisms isolated. ESBL: Extended Spectrum Beta Lactamase Producers; MDR: Multidrug Resistant.

\begin{tabular}{|l|c|c|}
\hline S. No & Mixed pathogens & No of patients \\
\hline 1 & E.coli ESBL and K. pneumoniae & 1 \\
\hline 2 & E. faecium and E. coli ESBL & 1 \\
\hline 3 & E. faecium and A. baumanii MDR & 1 \\
\hline 4 & E. faecalis and E. coli ESBL & 1 \\
\hline
\end{tabular}

Table 2: Distribution of mixed infections.

The patients were also treated with intravenous penicillin $10 \mathrm{U} /$ day. $11 / 12$ of the patients were discharged in stable condition with advise to follow up. The patient with above knee amputation succumbed to the infection in spite of the treatment. 


\section{Microbiological work up}

The tissue sample was subjected to Grams stain, aerobic culture, anerobic culture and also inoculated into thioglycolate broth. Grams stain showed large boxcar shaped Gram variable bacilli with no spores and neutrophils suggestive of Clostridium perfringens.

Culture on 5\% sheep Blood Agar (COS, biomeriux) showed double zone of beta hemolysis with no swarming after incubation at $37^{\circ} \mathrm{C}$ for 48 hours (Figure 2). A 5 - $\mu$ g metronidazole disk (Himedia) was placed on the blood agar surface and incubated for 48 to $72 \mathrm{~h}$ under anaerobic conditions, a zone of inhibition around the metronidazole disk was observed which indicates the presence of anaerobic bacteria. Aerotolerance was done on 5\% sheep blood agar and the plate was incubated in carbon dioxide pouch. Aerotolerance test showed no growth suggestive of the presence of anaerobic bacteria. Biochemical tests Indole and Urease were negative. Hence forth the culture was considered as $C$. perfringens.

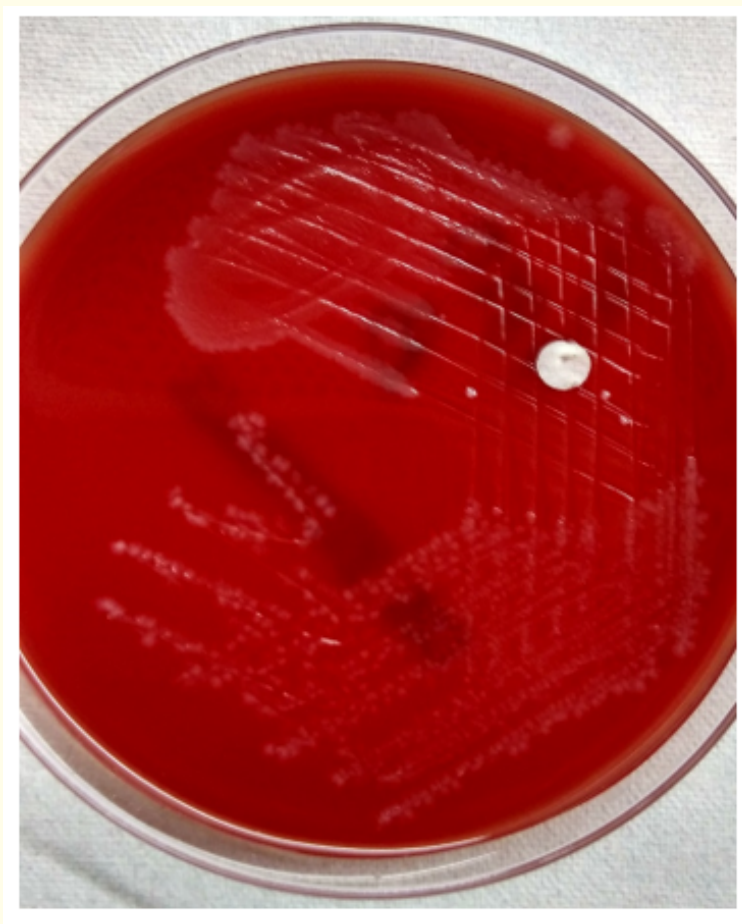

Figure 2: Culture on 5\% sheep Blood Agar (COS, biomeriux) showing double zone of beta hemolysis with no swarming and a zone of inhibition around 5 ug metronidazole disc after incubation at 370C for 48 hours.
Identification and susceptibility testing of all the aerobic bacterial pathogens was done by Vitek 2 Compact system (biomeriux) by using ID GN and N281panels for Gram negative organisms and ID GP and P628 panels for Gram positive organisms.

\section{Discussion}

French Microbiologist Louis Pasteur discovered Clostridium in 1860s. Clostridium species are Gram positive, strictly anaerobic, fermentative, spore-forming rods belonging to the phylum Firmicutes. They grow well at body temperature and can survive in adverse environment by the production of spores. They are ubiquitous in soil and appear as long thick rods with a bulge at one end [6].

The four clinically important species are C. tetani, $C$. botulinum, $C$. perfringens, and C. difficile, $C$. septicum that are responsible for causing diseases include tetanus, botulism, and gas gangrene and pseudomembranous colitis They secrete powerful exotoxins which is the virulence factor responsible for pathogenesis of disease [6].

Other species include $C$. butyricum, $C$. sordellii, $C$. bifermentans and $C$. sporogenes, Clostridium spp can be differentiated based on their cellular morphology, spores location biochemical activities, including saccharolysis and proteolysis gas liquid chromatography for fermentation products [6].

Gas gangrene is the infection caused by Clostridium perfringens which is an anaerobic, gram-positive bacillus inhabiting the human bowel and genitourinary tract [7]. It is commonly found in the gastrointestinal tract flora and a colonizer of the skin in the perirectal region. It was first isolated and identified in 1891 by William $\mathrm{H}$. Welch from the autopsy of a 38-year-old man, where gas bubbles were observed within infected blood vessels. The organism can easily invade wounds that come into contact with soil [7].

Clostridial myonecrosis or gas gangrene is very rare in pediatric population and is diagnosed more in adults [8]. Our study also showed the isolation of Clostridium perfringens from the adult population.

Clostridium perfringens is the most important of the species and accounts for $20-40 \%$ of all isolates [6] followed by $C$. septicum, $C$. novyi type A, C. histolyticum, and C. bifermentans [9]. In a study from Pondicherry Clostridium spp were reported in 56\% of cases [10]. The pathogen isolated in the present study was $C$. perfringens from all the cases. 
Gas gangrene is an invasive, fatal anaerobic infection that is often secondary to open fractures, deep wounds, and other injuries [4]. In the present study all the patients had fractures/crush injuries secondary to RTA. Five patients had compound fracture and seven had crush injuries.

The muscle and tissue are exposed to anaerobic bacteria like Clostridium spp after traumatic injury. Due to low oxygen tension [11] and adequate nutrients in the area of necrosis, the spores germinate and produce toxins like lecithinase [12-16]. The signs and symptoms of the infection include fever, with severe pain, redness of skin, muscle necrosis, accumulation of gas causing crepitance with a foul smelling discharge [4,12-16]. In the present study all the cases had exquisite pain, redness of skin, muscle necrosis clinically followed by foul smelling discharges from the wound. The patients with injury up to the thigh had gangrene of the thigh with crepitations.

The infection may develop hours to weeks after the initial trauma and inoculation. Necrotizing soft tissue infections occur with different degrees of severity and speed of progression involving the skin causing cellulitis, subcutaneous tissue to the muscle fascia leading to necrotizing fasciitis and muscle tissue causing Clostridial myonecrosis. In the present study the disease involved muscle tissue and effected progressively [17].

C. perfringens is associated with diverse environments including soils, food, sewage, and as a member of the gastrointestinal (GI) tract. Clostridium spp is mostly associated with development of traumatic gas gangrene but also can be associated with the nontraumatic spontaneous gas gangrene in patients with immunocompromised condition including malignancies and diabetes mellitus [18]. In the recent years there has been an increase incidence of this infection in road traffic accidents [19]. In the present study all the patients had sustained traumatic injury due to road traffic accidents. The probable source might be the soil containing spores of Clostridium which would have penetrated into the skin during the injury. None of the cases in the study were of spontaneous type.

Though Clostridium perfringens is recognized as the leading cause of gas gangrene, it can also be caused by non clostridial bacteria [20]. In addition, anaerobic streptococcal myositis also mimics gas gangrene [21]. In the present study there was no case of Streptococcal myositis documented.

Escherichia coli $(7 / 12)$ is the predominant aerobic organism in the present study of which 5/7 were ESBL producers. Similarly, in a study from Mumbai, India it was found that among aerobes isolated from the cases of gas gangrene along with anaerobes, Gram negative bacilli predominated (83.3\%) [22]. E. coli was the commonest aerobic isolate (23.3\%) in their study [22], and other studies [21]. Bessman and Wagner reported 49 cases of gas gangrene with myositis and soft tissue gas of which only one was caused by clostridial organisms [20].

Staphylococcus aureus has been described as being the single pathogen in two patients with rapidly progressing necrotizing fasciitis of the lower extremity and in a study of necrotizing fasciitis in eight children, Brook reported the presence of pure Streptococcus pyogenes in two patients and a mixed predominance of Pepto streptococcus spp., S. pyogenes, Bacteroides fragilis, C. perfringens, E. coli, and Prevotella spp. in the others [17]. Methicillin Resistant Staphylococcus aureus (MRSA) E. faecalis and E. faecium were the Gram positive pathogens that were isolated from the present study.

Treatment of histotoxic $C$. perfringens consists of penicillin G (to kill the organism), hyperbaric $\mathrm{O}_{2}$, administration of antitoxin, and debridement of infected areas [13]. In clinical situations that involve mixed infections, coverage against the other potential pathogens, aerobic as well as anaerobic, should be included. This can be achieved by choosing single therapy with an antimicrobial agent that possess wider coverage such as imipenem, meropenem, or by additional agents that cover other organisms with cefepime, an aminoglycoside or quinolone for Enterobacteriaceae, or a penicillinase-resistant penicillin, or Vancomycin for Staphylococcus aureus) [23]. Hassan., et al. and Wang., et al. [2,4] recommended definitive therapy to halt clostridial exotoxin production which includes Penicillin G plus Clindamycin. In the present study all the patients were treated with Penicillin G10 million units intravenously (IV) for every 4 hours and Clindamycin 600 - 900 mg IV every 8 hours along with metronidazole.

Definitive diagnosis is made by surgical exploration of the involved area and demonstration of the organism in tissue. In many cases of $C$. perfringens induced gas gangrene, radical amputation still remains the treatment of best choice [9]. All the patients in the present study undergone amputation and survived except one patient who succumbed.

The mortality rate is around $40-60 \%$ in studies from India and other countries $[24,25]$. In the present case series only one patient died. Other patients in the series survived probably due to the early

Citation: Kanne Padmaja., et al. "Clinical and Microbiological Spectrum of Clostridial Myonecrosis from a Tertiary Care Centre". Acta Scientific

Microbiology 3.9 (2020): 47-52. 
identification of the infection and appropriate management which is the need of the hour.

\section{Conclusion}

From this study we conclude that early clinical suspicion and microbiological diagnosis helps in the proper management of the Clostridial Myonecrosis patients. Careful and adequate treatment along with antibiotics and all other supportive treatments should be instituted immediately will avoid further deterioration and reduce the mortality in these patients.

\section{Bibliography}

1. Ying Z., et al. "Gas gangrene in orthopaedic patients". Case Reports in Orthopedics (2013): 942076.

2. Hassan SA., et al. "Frightening Resistant Clostridial Myonecrosis: A Case Report”. Cureus 11.4 (2019): e4539.

3. Dontchos BN., et al. "Spontaneous Clostridium perfringens myonecrosis: Case report, radiologic findings, and literature review". Radiology Case Report 8.3 (2015): 806.

4. Wang S and Liu L. "Gas gangrene following implant removal after the union of a tibial plateau fracture: a case report". BMC Musculoskeletal Disorders 19.1 (2018): 254.

5. "Clostridial toxins in the pathogenesis of gas gangrene". Amy E. Bryant, Dennis L. Stevens, in The Comprehensive Sourcebook of Bacterial Protein Toxins (Fourth Edition), (2015): 977-994.

6. Jousimies-Somer HR., et al. Wadsworth-KTL anaerobic bacteriology manual. 6th ed. Belmont, CA: Star Publishing (2002).

7. Van Bunderen CC., et al. "Clostridium perfringens septicaemia with massive intravascular haemolysis: a case report and review of the literature". Netherlands Journal of Medicine 68 (2010): 343-346.

8. García EK., et al. "Fulminant gas gangrene in an adolescent with immunodeficiency. Case report and literature review". Revista de la Facultad de Medicina 64.3 (2016): 555-559.

9. Stevens DL and Bryant AE. "The role of clostridial toxins in the pathogenesis of gas gangrene". Clinical Infectious Diseases 35 (2002): S93-S100.
10. Rao SR., et al. "An eight year bacteriological study of gas gangrene in Pondicherry". Indian Journal of Medical Microbiology 13.3 (1995): 151-154.

11. Buboltz JB and Murphy-Lavoie HM. "Gas Gangrene”. In: StatPearls. Treasure Island (FL): StatPearls Publishing (2020).

12. Kiu R and Hall LJ. "An update on the human and animal enteric pathogen Clostridium perfringens". Emerging Microbes and Infections 7.1 (2018): 141.

13. Jeffrey K Actor. Elseviers Integrated Review Immunology and Microbiology.

14. Rush DS., et al. "Prevention and management of common infections after trauma". In: Trauma. Mattox KL, Moore EE, Feliciano DV, editors. Norwalk: Appleton and Lange (1988): 223-226.

15. Caplan ES and Kluge RM. “Gas gangrene”. Archives of Internal Medicine 136 (1976): 788.

16. Nichols RL. "Gas gangrene and similar soft tissue infections". In: Conn R, editor. Current Diagnosis, 7th ed. Philadelphia: Saunders (1984): 149

17. Bowler PG., et al. "Wound microbiology and associated approaches to wound management". Clinical Microbiology Review 14.2 (2001): 244-269.

18. Pereira de Godoy JM., et al. "Mortality and diabetes mellitus in amputations of the lower limbs for gas gangrene: a case report". The International Journal of Lower Extremity Wounds 7.4 (2008): 239-240.

19. Udgaonkar US., et al. "Clinicobacteriological study of gas gangrene". Journal of the Indian Medical Association 88.1 (1990): 8-10.

20. Bessman AN and Wagner W. "Nonclostridial gas gangrene. Report of 48 cases and review of the literature". JAMA 233.9 (1975): 958-963.

21. Baradkar VP., et al. "Bacteriological study of clinically suspected cases of gas gangrene". Indian Journal of Medical Microbiology 17.3 (199): 133-134.

22. De A., et al. "Bacteriological studies of gas gangrene and related infections". Indian Journal of Medical Microbiology 21.3 (2003): 202-204. 
23. Fractures with Soft Tissue Injuries. Sanjeev Sabharwal, in Green's Skeletal Trauma in Children (Fifth Edition), (2015): 86-104.

24. Mathur P. "Infections in traumatised patients: a growing medico-surgical concern". Indian Journal of Medical Microbiology 26.3 (2008): 212-216.

25. Pereira de Godoy JM., et al. "Mortality and diabetes mellitus in amputations of the lower limbs for gas gangrene: a case report”. The International Journal of Lower Extremity Wounds 7.4 (2008): 239-240.

\section{Assets from publication with us}

- Prompt Acknowledgement after receiving the article

- Thorough Double blinded peer review

- Rapid Publication

- Issue of Publication Certificate

- High visibility of your Published work

Website: www.actascientific.com/

Submit Article: www.actascientific.com/submission.php

Email us: editor@actascientific.com

Contact us: +919182824667 